\title{
Plasmablasts walk the line
}

During an adaptive immune response, long-lived antibodyproducing plasma cells are generated in T cell-dependent germinal centres. Plasma cells subsequently localize to the lymph node medullary chords, but their migration to these sites has never been directly observed. A study in Immunity has now reported unique migratory behaviour for their precursors, plasmablasts; these cells traverse the lymph node in a linear manner and, surprisingly, do not require $\mathrm{Ga}_{\mathrm{i}}$-coupled receptor signalling to migrate.

The transcriptional repressor B lymphocyte-induced maturation protein 1 (BLIMP1) is crucial for plasma cell differentiation and is an early marker of plasma cell development. BLIMP1-yellow fluorescent protein (BLIMP1 ${ }^{\mathrm{YFP}}$ ) reporter mice expressing a transgenic $B$ cell receptor were therefore used to visualize plasma cell migration

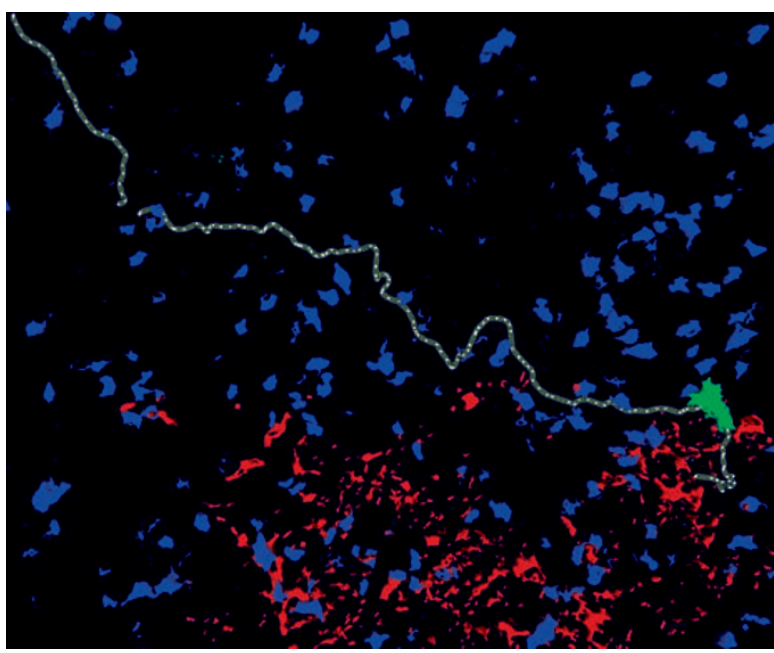

This image is taken from a time-lapse movie, which visualized a mouse popliteal lymph node during an immune response by two-photon intravital microscopy. A newly generated plasmablast (green) is emigrating from a germinal centre (red), with naive $\mathrm{B}$ cells (blue) in the follicle. The cell track (grey) shows a highly linear path over a period of 75 minutes. Image courtesy of D. R. Fooksman, New York University School of Medicine, New York, USA. @ 2010 Elsevier. patterns in lymph nodes. Following immunization with specific antigen, $\mathrm{YFP}^{+}$cells mainly aggregated in the medulla of the lymph node but could also be found in the B and $\mathrm{T}$ cell zones and at the border of germinal centres. By contrast, naive $\mathrm{B}$ cells were predominantly localized in B cell follicles.

When the migratory patterns of the different populations were observed in situ using time-lapse two-photon intravital microscopy, $\mathrm{YFP}^{+}$cells in the medullary chords were found to be mainly stationary, but $\mathrm{YFP}^{+}$cells in the follicles were highly motile. Naive B cells showed random, winding patterns of motion, but migration of extramedullary $\mathrm{YFP}^{+}$cells was steady and linear. Despite having decreased migratory speeds compared with naive B cells, $\mathrm{YFP}^{+}$cells had higher rates of displacement, consistent with these cells moving in a constant, linear manner. However, $\mathrm{YFP}^{+}$cells did not show any evidence of directional movement, suggesting that plasmablasts do not use preformed chemotactic gradients to travel to the medulla.

To more closely examine the role of chemokines in plasmablast migration, the authors treated cells with pertussis toxin (PTX), which blocks $\mathrm{Ga}_{\mathrm{i}}$-mediated signalling and prevents most chemokine-directed movement. Strikingly, whereas PTX treatment inhibited naive $\mathrm{B}$ cell migration, PTX-treated $\mathrm{YFP}^{+}$cells continued to crawl in a persistent, linear manner. Further experiments using the inhibitor FTY720 indicated that plasmablast migration in the lymph node was also independent of sphingosine-1-phosphate (S1P) receptor signalling. The authors next assessed the role of the adhesion molecule intercellular adhesion molecule 1 (ICAM1) in plasmablast migration. Both $\mathrm{YFP}^{+}$and naive B cells migrated on ICAM1-coated glass surfaces, but only naive B cells required $\mathrm{Ga}_{\mathrm{i}}$-signalling for this movement. In addition, naive $B$ cells and plasmablasts had different migratory behaviour on the ICAM1-coated surfaces; naive $B$ cells showed frequent detachment and reattachment to the substrate, but $\mathrm{YFP}^{+}$cells migrated in a steady, amoeboid manner.

Interestingly, in assays carried out in vivo and in vitro, the authors reported an inverse correlation between cell speed and cellular YFP intensity as BLIMP1 expression increases during plasma cell maturation; this suggests that plasmablast migration and arrest is cell autonomous. Finally, although plasmablasts could migrate independently of $\mathrm{Ga}_{\mathrm{i}}$-mediated signalling, PTX treatment at early time points following immunization decreased plasma cell accumulation in the medulla; however, YFPhi cells were enriched in the $\mathrm{B}$ and $\mathrm{T}$ cell zones following PTX treatment, suggesting that plasmablast maturation is also cell autonomous.

The authors propose a model in which plasmablasts seek out medullary cords by undertaking random, linear migration paths. Both the migration and maturation of plasmablasts seem to be cell autonomous, but chemokine receptor signalling may be required for plasma cell retention in the medulla.

Yvonne Bordon

ORIGINAL RESEARCH PAPER Fooksman, D. R. et al. Development and migration of plasma cells in the mouse lymph node. Immunity $8 \mathrm{Jul} 2010$ (doi:10.1016/j.immuni.2010.06.015) 